\title{
CONF-931118-6.8
}

Note: This is a preprint of a paper submitted for publication. Contents of this paper should not be quoted or referred to without permission of the author(s).

For publication in Growth, Processing, and Characterization of Semiconductor Heterostructures, ed. by G. Gumbs, S. Luryi, B. Weiss, and G. W. Wicks, Materials Research Society, Pittsburgh, Pennsylvania,

(Fall Meeting of the Materials Research Society, Boston, Massachusetts,

November 29-December 3, 1993

\section{STRUCTURAL CHARACTERIZATION OF SEMICONDUCTOR HETEROSTRUCTURES BY ATOMIC RESOLUTION Z-CONTRAST IMAGING AT $300 \mathrm{KV}$}

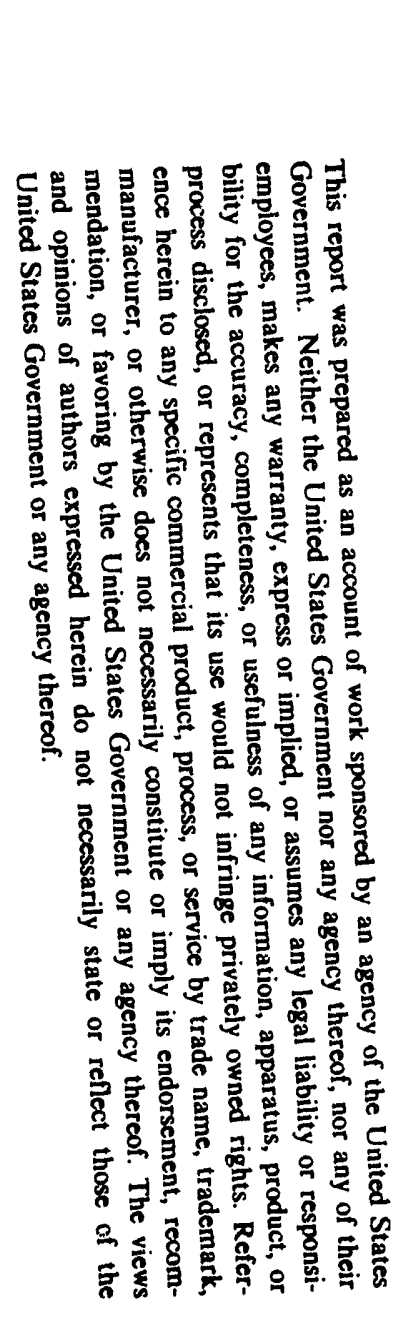

A. J. McGibbon and S. J. Pennycook

Solid State Division

Oak Ridge National Laboratory

Oak Ridge, Tennessee 37831-6031

Z. Wasilewski

Institute for Microstructural Sciences

National Research Council of Canada

Ottawa, Ontario K1A 0R6, Canada

"The submitted manuscript has been authored by a contractor of the U.S. Govemment under contract No. DE-AC05-840R2 1400.

Accordingly, the U.S. Government retains :

nonexclusive, royalty-free license to publish or

reproduce the published form of this

contribution, or allow others to do 20 , for U.S.

Government purposes."

SOLID STATE DIVISION

OAK RIDGE NATIONAL LABORATORY

Managed by

IARTIN MARIETTA ENERGY SYSTEMS, INC.

under

Contract No. DE-AC05-84OR21400

with the

U.S. DEPARTMENT OF ENERGY

Oak Ridge, Tennessee

December 1993

MASTER 


\title{
STRUCTURAL CHARACTERIZATION OF SEMICONDUCTOR HETEROSTRUCTURES BY ATOMIC RESOLUTION Z-CONTRAST IMAGING AT 300KV
}

\author{
A. J. McGIBBON*, S. J. PENNYCOOK* AND Z. WASILEWSKI** \\ *Solid State Division, Oak Ridge National Laboratory, Oak Ridge, TN 37831-6031, USA \\ **Institute for Microstructural Sciences, National Research Council of Canada, Ottawa, \\ Ontario K1A 0R6, Canada
}

\begin{abstract}
By applying the technique of Z-contrast imaging to the study of a GaAs/AlGaAs multilayer using a newly developed $300 \mathrm{kV}$ scanning transmission electron microscope, we show that it is possible to directly observe the interlocking group III and group V sub-lattices on a column-bycolumn level. In addition to the direct observation of structural polarity in the $\langle 110\rangle$ orientation, we show that, by using a maximum entropy approach to image processing, the experimentally acquired data can provide direct information on interface structures at atomic resolution.
\end{abstract}

\section{INTRODUCTION}

III-V semiconductor heterostructures are technologically important materials whose macroscopic properties rely strongly on the precise nature of the atomic arrangements within. It is highly desirable therefore, to obtain directly interpretable, compositionally sensitive images of interface structures in these materials at atomic resolution. Until now, microstructural investigations of compound semiconductors in the electron microscope have involved techniques such as chemical lattice imaging (1), high resolution electron microscopy (HREM) (2) and convergent beam electron diffraction (CBED) (3). However, interpretation of data acquired using such techniques in terms of the actual atomic structure can be problematical.

In this paper, we discuss the application of Z-contrast imaging $(4,5)$ in a recently developed $300 \mathrm{kV}$ scanning transmission electron microscope (STEM) to the study of the sub-lattice in III$\mathrm{V}$ semiconductors. The technique, previously carried out at accelerating voltages of $100 \mathrm{kV}$, enables the acquisition of direct, compositionally sensitive images of material structures at high spatial resolution. Here, we show that the increased spatial resolution facilitated by operating at $300 \mathrm{kV}$ enables the observation of nearest-neighbor separations in III-V semiconductor specimens oriented along the $\langle 110\rangle$ zone-axis. Furthermore, the elemental sensitivity of Zcontrast imaging makes it possible to discriminate between the group III and group V sites. Emphasis here is given to the study of a GaAs/AlGaAs multilayer. Conclusions are drawn on the spatial resolution and atomic number sensitivity of the technique and, with the application of a maximum entropy approach to image analysis (6), the way in which the acquired image can be used to give detailed atomic resolution information without recourse to pre-conceived models of the original structure.

\section{Z-CONTRAST IMAGING IN A 300kV STEM}

A schematic diagram outlining the technique of Z-contrast imaging in STEM is given in figure 1 . In a STEM, a high current density, finely ' $x$ cused electron probe is scanned in a raster pattern across the specimen of interest. Z-contrast imaging is carried out by recording the integrated intensity of transmitted electrons scattered through high angles at each probe position. The key feature of this technique in the analysis of crystalline specimens is that high angle contributions are dominated by incoherent, thermal diffuse scattering, eliminating coherent contributions between individual columns in the material (7). Consequently, when examining a specimen oriented along a zone axis, the resultant image is a map of the columnar scattering 
intensity, the resolution of which is primarily limited by the size of the electron probe. Furthermore, the variations in image intensity approach that of the $Z^{2}$ dependence of unscreened Rutherford scattering and so it is possible to directly observe variations in columnar intensity which are in turn related to changes in mean atomic number from column to column. Unlike phase contrast techniques employed in HREM (2) in which image contrast reversals can occur as a function of electron beam defocus, Z-contrast imaging is an incoherent imaging technique, and as such the results obtained at the Scherzer defocus condition (8) can be interpreted directly in terms of the position, relative contrast and spacing of the atomic columns in the specimen.

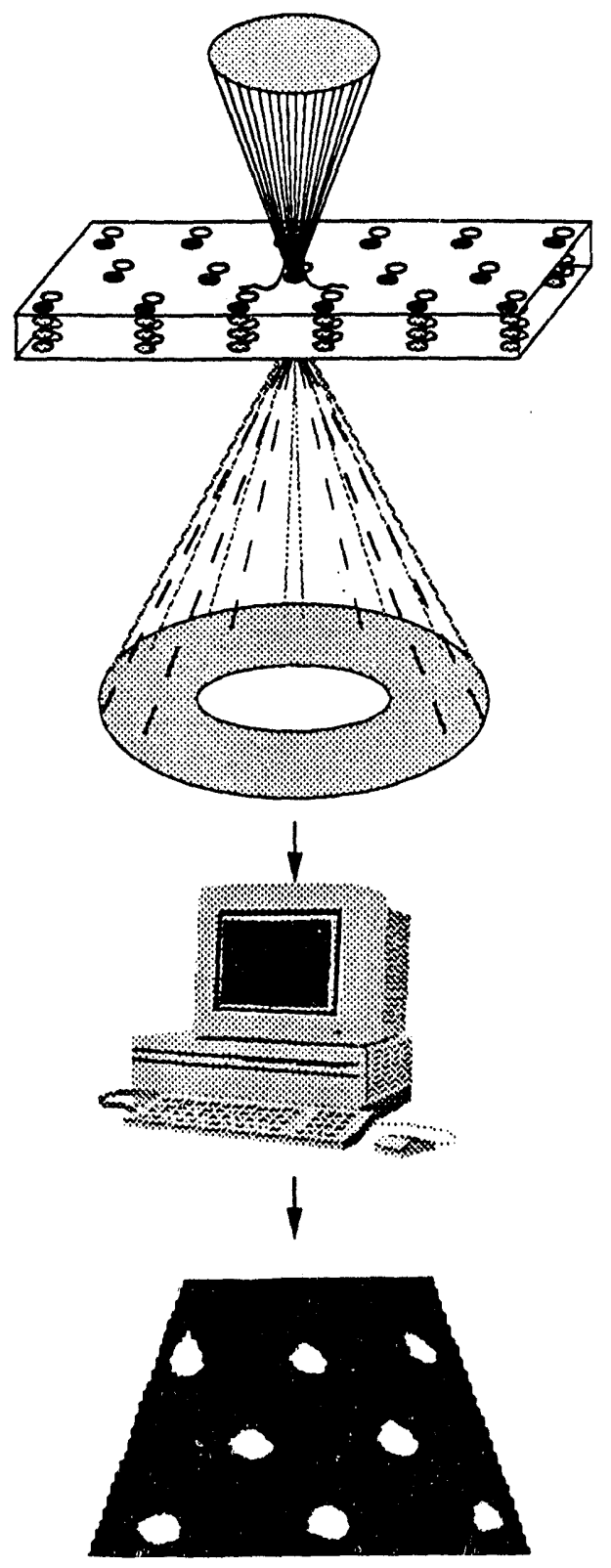

Incident electron probe

Semiconductor cross-section

High-angle thermal diffuse scattering

Annular Detector

Acquisition and processing

Atomic resolution Z-contrast image

Figure 1. A schematic diagram depicting the formation of an atomic resolution image using Zcontrast imaging in a $300 \mathrm{kV}$ STEM. 
The microscope used in the experiments described here was a VG Microscopes HB603U STEM. When operating at $300 \mathrm{kV}$, this microscope can provide an electron probe full-width half-maximum (FWHM) of $1.3 \AA$ at Scherzer defocus (total current $\sim 0.1 \mathrm{n} A$ ), compared to a value of $2.2 \AA$ at $100 \mathrm{kV}$. The material of interest was a GaAs/Al0.1Ga0.9As multilayer consisting of alternate layers of $10 \mathrm{~nm} \mathrm{Al} 0.1 \mathrm{Ga} 0.9 \mathrm{As}$ and $30 \mathrm{~nm} \mathrm{GaAs}$ and grown on a GaAs substrate by molecular beam epitaxy (MBE). For observation in the STEM, a thin film $(\sim 100 \AA)$ cross-sectional specimen in the $<110>$ orientation was prepared using ion beam thinning as the final stage of preparation. Images were acquired using an annular detector possessing an inner collection angle of $\sim 40 \mathrm{mrad}$. To enable the attainment of atomic resolution, specimen drift and mechanical instabilities were minimized. The total acquisition time for an atomic resolution image (covering 10 unit cells in area) was 20 seconds. No irradiation damage of the material was observed at any time during the experiment. The nature of STEM enables all images to be acquired digitally, allowing detailed post-acquisition analysis of the original data. The $\mathrm{GaAs} / \mathrm{AlGaAs}$ system is ideally suited to initial studies using the technique described here, as both the nearest neighbor separation in the $\langle 110\rangle$ orientation $(1.41 \AA)$ and the relative change in intensity between, for example, columns of $\mathrm{Ga}$ and $\mathrm{As}$ ( $13.3 \%$ at the full $\mathrm{Z}^{2}$ value) is within the achievable limits of the system.

\section{DIRECT OBSERVATION OF THE SUB-LATTICE IN GaAs}

As a measure of both the spatial resolution and Z-sensitivity capabilities of the technique described here, Z-contrast images of regions in the GaAs substrate were acquired, and one such image is shown in figure 2. It can be seen from figure 2 that it is possible to resolve the individual columns which form the column pairs or 'dumbbells' that occur in the $\langle 110\rangle$ orientation. Furthermore, the recorded intensity in the lower half of each dumbbell is uniformly higher than the upper half. This can be seen more clearly in figure 3(a) by selecting a region of the image for further analysis. In figure 3, for the purposes of discriminating between relatively small changes in intensity, a $\gamma$-correction of 0.5 was applied to all images. Without the application of noise filtering techniques, figure 3(a) clearly shows that using Z-contrast imaging at $300 \mathrm{kV}$ it is possible to directly observe the structural polarity of GaAs in the $<110>$ orientation.
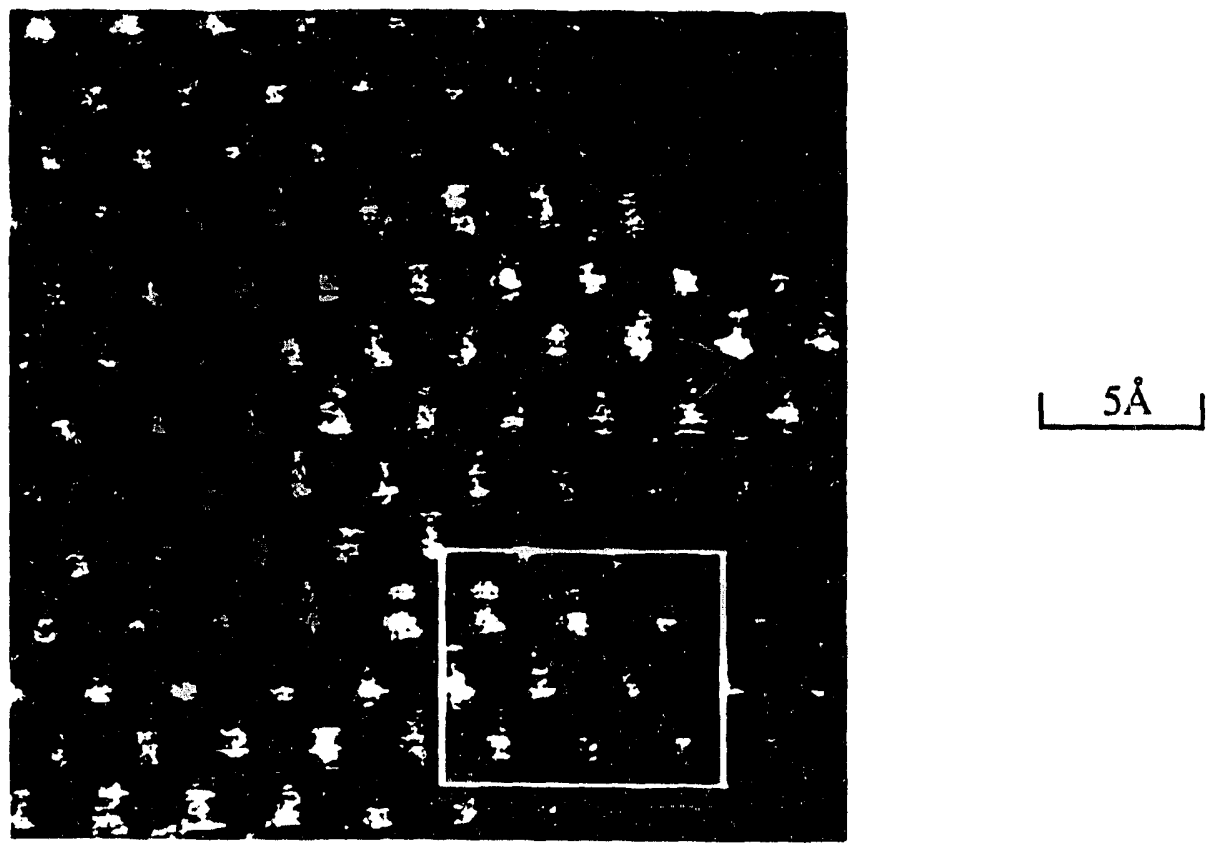

Figure 2. As-acquired high spatial resolution Z-contrast image of a region of GaAs substrate. Box shows region of interest in figure 3. 
Image quality enhancement of as-acquired data without the loss of spatial or compositional information can be easily obtained by the application of simple image processing techniques such as median filtering (figure $3 \mathrm{~b}$ ). However, as stated previously, Z-contrast images possess information on the variation in columnar scattering intensity across the region of interest. As a route, therefore, to retrieving such information on a quantitative basis, a maximum entropy approach to image processing (6) is being pursued. Using this technique, a 'most likely' image reconstruction is obtained given a particular incident probe function. Provided that an accurate description of the electron probe can be obtained, it is possible to describe the specimen function as an array of point scatterers with intensities related to the high-angle scattering cross-

(a)

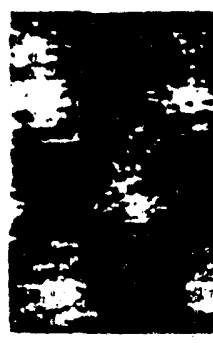

(b)

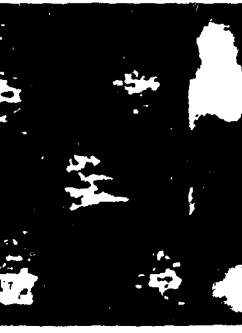

(c)

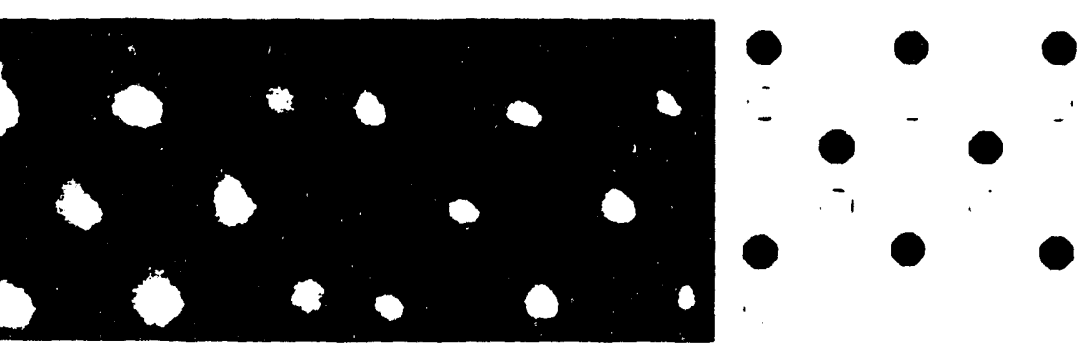

(d)

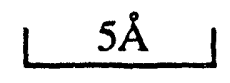

Figure 3. Region of GaAs shown as (a) an as-acquired image, (b) median filtered image of (a), (c) maximum entropy reconstruction of (a), and (d) schematic diagram of structure. The group $\mathrm{V}$ site occupies the lower column of each dumbbell.

section of each column, thus providing quantitative information on a column-by-column level (9). However, for the purposes of this experiment, the incident probe used was a Lorentzian distribution possessing a FWHM of $1.3 \AA$. The result of 20 iterations of the maximum entropy program using such a probe is shown in figure $3(\mathrm{c})$. The high quality of such an image in comparison to a schematic diagram of the actual atomic structure given in figure $3(\mathrm{~d})$ emphasizes the potential of the technique in the analysis of III-V semiconductors.

\section{ANALYSIS OF INTERFACE STRUCTURES}

Having demonstrated the power of atomic resolution Z-contrast imaging in STEM, we now consider the application of the technique to the study of a GaAs/Al0.1Ga0.9As interface. A series of Z-contrast images (without contrast enhancement) of the multilayer specimen at increasing magnifications is shown in figure 4. It is clear from this figure that, despite relatively small changes in the mean atomic number between each layer, intensity changes that are attributable to the presence of $\mathrm{Al}$ are readily observed. By extracting a small area from the image of highest magnification, it is possible to investigate in detail the interface structure (figure 5). In a manner similar to the as-acquired image of the GaAs substrate, figure 5(a) clearly shows that the stringent requirements for spatial resolution and Z-sensitivity in the analysis of III-V semiconductors have been achieved. A 20-iteration maximum entropy reconstruction of this

image is shown in figure 5(b). Qualitatively, it can again be seen that the group V site occupies the lower half of each dumbbell, and that the As sub-lattice is continuous throughout the interface region. Variations in the intensity of the As columns in the maximum entropy image may be attributable to inconsistencies between the Lorentzian probe approximation and that of the actual probe current density distribution used in the experiment. 
However, the relative intensity of the upper column of each dumbbell varies significantly across the interface, corresponding to the substitution of $\mathrm{Al}$ atoms for those of $\mathrm{Ga}$ in the group III sites. Further investigation and discussion of this image in terms of columnar scattering intensities would require a more detailed knowledge of the current density distribution of the incident electron probe.

(a)

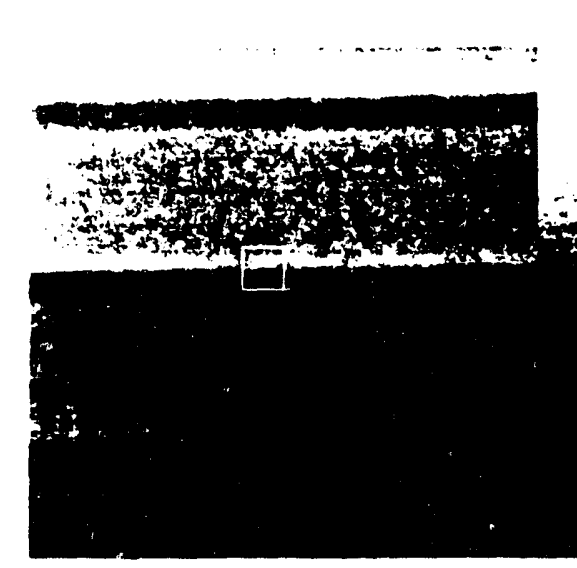

$30 \mathrm{~nm}$ (b)

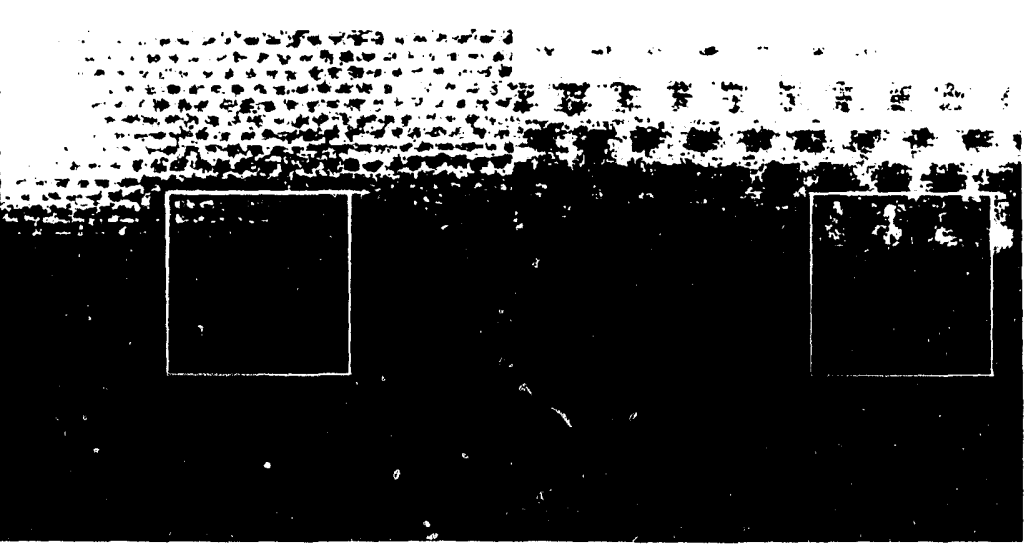

(c)

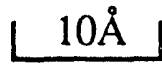

(a)

(b)

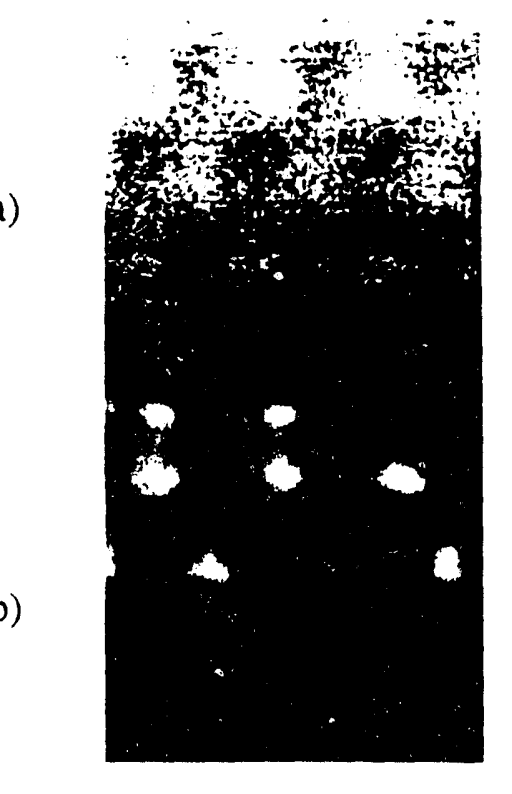

$3 \mathrm{~nm}$

Figure 4. Z-contrast images of a GaAs/Al0.1 Ga0.9As multilayer acquired at (a) low, (b) intermediate and (c) high magnification. Box in (c) shows region of interest in figure 5.

Figure 5. Interface region between a layer of GaAs (upper half) and $\mathrm{Al}_{0.1} \mathrm{Ga} 0.9 \mathrm{As}$ (lower half) shown as (a) an as-acquired image and (b) maximum entropy reconstruction of (a). The group V site occupies the lower column of each dumbbell.

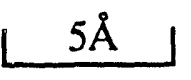

\section{DISCUSSION AND CONCLUSIONS}

By observation of the structural polarity of GaAs in the $<110>$ orientation, we have clearly demonstrated that $Z$-contrast imaging in a $300 \mathrm{kV}$ STEM can provide direct, compositionally sensitive information on III-V semiconductor specimens on the atomic scale. Using this technique, it is now possible to investigate, for example, the effect of structural polarity and atomic ordering (10) on the atomic arrangements at interfaces in compound semiconductors. In 
addition, by applying the technique of maximum entropy to image analysis, detailed quantitative information on an atomic scale may be possible. Such information can have a profound effect on the understanding of materials growth processes and the macroscopic properties of fabricated structures.

\section{ACKNOWLEDGMENTS}

We would like to thank T. C. Estes, J. T, Luck and S. L. Carney for technical assistance. This research was sponsored by the Division of Materials Sciences, US Department of Energy, under contract No. DE-AC05-840R21400 with Martin Marietta Energy Systems, Inc., and supported in part by an appointment to the Oak Ridge National Laboratory Postdoctoral Research Program administered by the Oak Ridge Institute for Science and Education.

\section{REFERENCES}

1. A. Ourmazd, F. H. Baumann, M. Bode and Y. Kim, Ultramicroscopy 34237 (1990)

2. D. J. Smith, L. A. Bursill and G. J. Wood, Ultramicroscopy 1619 (1985)

3. J. Tafto and J. C. H. Spence, J. Appl. Cryst. 1560 (1982)

4. S. J. Pennycook and D. E. Jesson, Ultramicroscopy 3714 (1991)

5. S. J. Pennycook and D. E. Jesson, Acta Metall. Mater. 40 S149 (1992)

6. S. F. Gull and J. Skilling, IEEE Proc. 131F 646 (1984)

7. D. E. Jesson and S. J. Pennycook, Proc. R. Soc. Lond. A 441261 (1993)

8. O. Scherzer, J. Appl. Phys. 2020 (1949)

9. S. J. Pennycook, D. E. Jesson, M. F. Chisholm, A. G. Ferridge and M. J. Seddon, Proc. 10th Pfefferkorn Conf. on Signal Processing, Cambridge, U.K. (1991)

10. T. S. Kuan, T. F. Kuech, W. I. Wang and E. L. Wilkie, Phys. Rev. Lett. 54201 (1985) 
